PREPARED FOR THE U.S. DEPARTMENT OF ENERGY, UNDER CONTRACT DE-AC02-76CH03073

PPPL-3475

PPPL-3475

UC-70

lon Heating in the Field-reversed Configuration (FRC)

by Rotating Magnetic Fields (RMF) near Cyclotron Resonance

by

Samuel A. Cohen and Alan H. Glasser

July 2000

$=$ PPPL

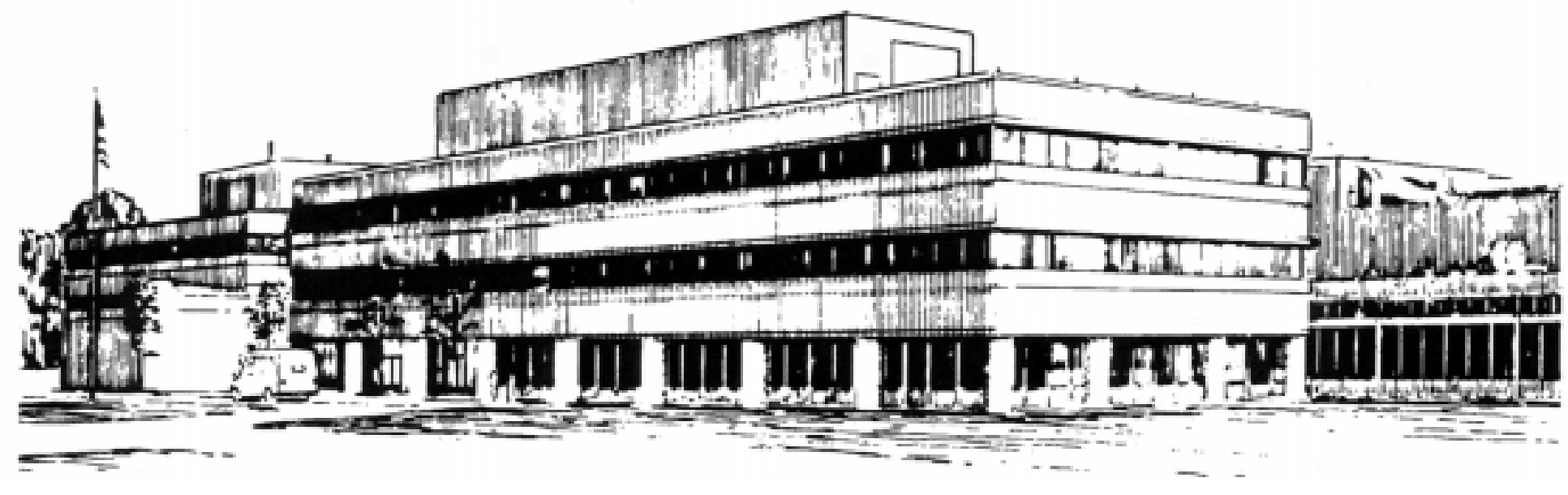

PRINCETON PLASMA PHYSICS LABORATORY PRINCETON UNIVERSITY, PRINCETON, NEW JERSEY 


\section{PPPL Reports Disclaimer}

This report was prepared as an account of work sponsored by an agency of the United States Government. Neither the United States Government nor any agency thereof, nor any of their employees, makes any warranty, express or implied, or assumes any legal liability or responsibility for the accuracy, completeness, or usefulness of any information, apparatus, product, or process disclosed, or represents that its use would not infringe privately owned rights. Reference herein to any specific commercial product, process, or service by trade name, trademark, manufacturer, or otherwise, does not necessarily constitute or imply its endorsement, recommendation, or favoring by the United States Government or any agency thereof. The views and opinions of authors expressed herein do not necessarily state or reflect those of the United States Government or any agency thereof.

\section{Availability}

This report is posted on the U.S. Department of Energy's Princeton Plasma Physics Laboratory Publications and Reports web site in Calendar Year 2000. The home page for PPPL Reports and Publications is: http://www.pppl.gov/pub_report/

DOE and DOE Contractors can obtain copies of this report from:

U.S. Department of Energy

Office of Scientific and Technical Information

DOE Technical Information Services (DTIS)

P.O. Box 62

Oak Ridge, TN 37831

Telephone: (865) 576-8401

Fax: (865) 576-5728

Email: reports@adonis.osti.gov

This report is available to the general public from:

National Technical Information Service

U.S. Department of Commerce

5285 Port Royal Road

Springfield, VA 22161

Telephone: $1-800-553-6847$ or

(703) $605-6000$

Fax: (703) 321-8547

Internet: http://www.ntis.gov/ordering.htm 


\title{
Ion heating in the field-reversed configuration (FRC) by rotating magnetic fields (RMF) near cyclotron resonance
}

Samuel A. Cohen, Princeton University, Plasma Physics Laboratory, Princeton, NJ 08543 Alan H. Glasser, PO Box 1663, Los Alamos National Laboratory, Los Alamos, NM 87545

\begin{abstract}
The trajectories of ions confined in a Solov'ev FRC equilibrium magnetic geometry and heated with a small-amplitude, odd-parity rotating magnetic field, have been studied with a Hamiltonian computer code. When the RMF frequency is in the ion-cyclotron range, explosive heating occurs. Higher-energy ions are found to have betatron-type orbits, preferentially localized near the FRC midplane. These results are relevant to a compact magnetic-fusion-reactor design.
\end{abstract}

As a fusion reactor, the field-reversed configuration ${ }^{1}$ (FRC) has attractive features, notably a linear magnet geometry and high- $\beta$ operation $(\beta \equiv$ plasma pressure/magnetic field pressure). The latter is essential for burning advanced, aneutronic fuels, which would considerably ease important engineering and environmental problems. ${ }^{2}$ Several physics challenges remain for the $\mathrm{FRC}$ to be developed into a practical fusion device: adequate energy confinement, stability against the internal tilt mode, ${ }^{3}$ and practical methods to sustain the plasma configuration and heat the ions ${ }^{4}$ to fusion-relevant temperatures, suitable for the relatively compact $\mathrm{FRC} .^{5}$

This paper examines the use of a new class of rotating magnetic fields, those of oddparity about the midplane ${ }^{6}$ to heat ions. Odd-parity modes are used because they are thought to improve energy confinement by maintaining field closure.

Rotating magnetic fields (RMFs) have been successfully used, particularly in rotamak devices, ${ }^{7,8}$ to make plasma, drive toroidal current, and obtain field reversal. In studies of current drive in rotamaks, ${ }^{9}$ usually only electron motion has been considered because the RMF frequency, $\omega_{R}$, has been chosen to be large compared to the ion cyclotron frequency in the RMF, ${ }_{R} \omega_{c i} \equiv q_{i} B_{R} / M_{i} c$, with $q_{i}$ and $M_{i}$ the charge and mass of the ion and $B_{R}$ the RMF amplitude. The ion motion was thus assumed to be a mere quiver, not of practical importance. However, to minimize circulating power, $B_{R}$ in an FRC reactor must be much weaker than the main axial field, $B_{a}$. Then the condition $\omega_{R} \gg{ }_{R} \omega_{c i}$ does not preclude $\omega_{R} \sim \omega_{c i} \equiv q_{i} B_{a} / M_{i} c$ the ion cyclotron frequency in the main axial field. This broad resonance is shown here to be an effective ion heating mechanism.

Previous studies of ion motion in RMFs have missed this effect because they were in different regimes of frequency, field strength, and duration. Ion motion in infinitely long ${ }^{10}$ and finite length ${ }^{11}$ FRCs with even-parity, $\omega_{R} \gg \omega_{c i}$, RMFs has been analyzed for durations up to 250 gyro-periods. These relatively short-time-scale studies showed no ion heating. In marked contrast, we have examined effects of odd-parity RMFs on ion orbits in an FRC for which $\omega_{R} \sim \omega_{c i}$ and find conditions, for laboratory-scale experiments and reactors, under which ions are explosively heated to energies sufficiently high to be fusion-relevant. For $B_{R} / B_{a} \sim 10^{-3}$, typically more than 1000 gyro-orbits are necessary for 
appreciable heating. We note, in passing, that stability against the tilt mode is improved by energetic ions ${ }^{12,13}$ and perhaps by the RMF itself. ${ }^{14}$

The physical mechanism for this heating has limited similarities to ion cyclotron resonance heating. ${ }^{15,16}$ The link is weak because the full 3D geometry with steep gradients, ${ }^{17}$ characteristic of the FRC, does not provide a distinct resonance region. Also different from ICRF heating are the purely inductive nature of the FRC's electric field, the global departure from adiabaticity, and the relatively weak plasma response due to the assumed synchronous rotation of the electrons with the fully penetrated RMF.

RMF penetration is found ${ }^{9}$ to be controlled by the ratio of two dimensionless numbers, $\gamma$ (the ratio of electron cyclotron frequency in the RMF field to the electron-ion collision frequency) and $\lambda$ (the ratio of the separatrix radius to the classical skin depth). When $\gamma / \lambda>1.2$, good penetration is predicted. For the reference FRC (RFRC) described below $\gamma / \lambda \sim 5$ and full RMF penetration is expected.

There is also a weak similarity to Fermi acceleration ${ }^{18}$ (FA), in which a particle in a box gains energy by colliding with an oscillating wall. When an odd-parity RMF is applied to a "plasma-less" FRC, periodic axial and radial expansion and contraction of the closed field lines (flux surfaces) occur, ${ }^{6}$ similar to moving walls in FA. However, this picture is too simplistic. First, the "moving magnetic walls" of the FRC are soft compared with the standard FA hard-wall models; particles gain energy throughout the volume, not just at the boundaries. Second, particle acceleration is due to the time-varying $\mathbf{E}$ and $\mathbf{B}$ fields and may be perpendicular or parallel to the moving magnetic wall. Finally, the FRC-RMF geometry is fully 3D, not 1D, hence no KAM surfaces should exist and Arnold diffusion of energy and Levy trajectories are expected. ${ }^{19}$

Our study is based on following single-particle trajectories in the fields of the FRC and RMF. This is appropriate if the collisionality is sufficiently low. A low-collisionality criterion is that the ratio of system size, $r_{s}$, to collision length, $\lambda$, be small, i.e., $r_{s} / \lambda<$ 0.1. For Coulomb collisions, this corresponds to $E_{m}^{2} / n_{i} r_{s}>10^{-11}$, where $r_{s}(\mathrm{~cm})$ is the separatrix radius of the FRC, $E_{m}(\mathrm{eV})$ is the minimum ion energy, and $n_{i}\left(\mathrm{~cm}^{-3}\right)$ is the plasma density. At $n_{i}=10^{14} \mathrm{~cm}^{-3}, E_{m}=100 \mathrm{eV}$ for a $10-\mathrm{cm}$ radius device. The explosive heating described here allows particles to make a quick transition from marginally collisional to fully collisionless.

The confining magnetic field of the FRC is described inside the separtrix as an elongated Hill vortex,

$$
r A_{\phi}=\psi_{0}\left(\frac{r^{2}}{r_{s}^{2}}\right)\left(1-\frac{r^{2}}{r_{s}^{2}}-\frac{z^{2}}{z_{s}^{2}}\right), \quad B_{r}=\frac{1}{r} \frac{\partial\left(r A_{\phi}\right)}{\partial z}, \quad B_{z}=-\frac{1}{r} \frac{\partial\left(r A_{\phi}\right)}{\partial r}
$$

with $A_{\phi}$ the azimuthal vector potential, $\psi_{0}=B_{a} r_{s}^{2} / 2$ and $B_{a}$ the field strength at the midpoint. To be fusion-relevant, the final ion energy must exceed $\sim 10 \mathrm{keV}$ for $\mathrm{D}-\mathrm{T}$ and $\sim 40 \mathrm{keV}$ for $\mathrm{D}-\mathrm{He}_{3} .{ }^{20}$ This imposes constraints on the size and field strength. For the sample calculations shown later, we fix $B_{a}=20 \mathrm{kG}$. At this field, deuterium ions have $\omega_{c i} \sim 10^{8} \mathrm{~Hz}$ and $3.6 \mathrm{MeV} \alpha$ particles (fusion-reaction products) have a $10 \mathrm{~cm}$ gyroradius. We assume the FRC to be elongated, $\kappa \equiv z_{s} / r_{s}=5$, due to the favorable affect on tilt 
stability and, as show below, to improve heating. We designate this as the reference FRC (RFRC). The vacuum field outside the separatrix ${ }^{21}$ used for the RFRC creates magnetic cusps near the $\mathrm{x}$-points at elevations of $z \sim \pm 52 \mathrm{~cm}$. Low confining field strength near the $\mathrm{x}$-points (at $\pm z_{s}$ ) and in the cusp region outside the separatrix creates potential loss channels for ions. The vacuum vector potentials for the odd-parity RMF are given by

$$
\left\{A_{r}, A_{z}, A_{\phi}\right\}=\frac{2 B_{R}}{k}\left\{I_{0}(\xi) \cos k z \sin \psi, I_{1}(\xi) \sin k z \sin \psi, I_{0}(\xi) \cos k z \cos \psi\right\}
$$

with $\psi \equiv \phi-\omega_{R} t, \xi \equiv k r, k=l \pi / \kappa r_{s}$ is the wave number of the RMF, $l$ is the axial mode number, and the $I_{m}$ are modified Bessel functions. Near $z=0$, the axial component $A_{z}$ and the electric field due to its time derivative are small compared to the corresponding radial and azimuthal components.

Using a computer code RMF_1.13, we numerically integrate the six nonlinear differential equations comprising Hamilton's equations,

$$
\begin{aligned}
& H=\frac{1}{2 M_{i}}\left[\left(p_{r}-\frac{q_{i}}{c} A_{r}\right)^{2}+\left(p_{z}-\frac{q_{i}}{c} A_{z}\right)^{2}+\frac{1}{r^{2}}\left(p_{\phi}-\frac{q_{i}}{c} r A_{\phi}\right)^{2}\right]+q_{i} \varphi \\
& \dot{r}=\frac{\partial H}{\partial p_{r}}, \quad \dot{z}=\frac{\partial H}{\partial p_{z}}, \quad \dot{\phi}=\frac{\partial H}{\partial p_{\phi}}, \quad \dot{p}_{r}=-\frac{\partial H}{\partial r}, \quad \dot{p}_{z}=-\frac{\partial H}{\partial z}, \quad \dot{p}_{\phi}=-\frac{\partial H}{\partial \phi},
\end{aligned}
$$

with an adaptive multistep method. ${ }^{22}$ We set the electrostatic potential to $\varphi=0$; such a potential has been predicted, ${ }^{23}$ but has not been verified. Since $H$ depends on $\phi$ and $t$ only through $\psi$, it follows that the transformed Hamiltonian $K \equiv H-\omega p_{\phi}$ is conserved. This is used to monitor the accuracy of numerical integration; it is conserved to a relative tolerance $\leq 10^{-3}$ in all runs reported here, even while some of these runs require $\sim 10^{6}$ integrator steps.

In a typical code run, a 100-eV deuteron is initialized at a position inside the RFRC's separatrix. Other initial parameters are the angles of the particle's velocity, and the phase, frequency, and amplitude of the RMF. Deuterons with $100 \mathrm{eV}$ perform cyclotron orbits, unless they are very close to the O-point null line, in which case they may perform null-linecrossing betatron orbits. In an FRC, ion cyclotron orbits drift in one toroidal direction, antiparallel to the FRC current, while betatron orbits drift in the opposite direction. (An intermediate class of null-line-crossing orbits exists. These, termed figure- 8 orbits, ${ }^{24}$ may drift in either direction.) The sign of $\omega_{R}$ is positive when the RMF rotates in the direction of the ion cyclotron drift.

These types of orbit-following calculations are similar to those performed for ions and electrons in the magnetotail. ${ }^{25}$ Complicated dynamics, such as Speiser orbits, ${ }^{26}$ have been observed, even though the calculations were only $2 \mathrm{D}$ and time-dependent fields, such as a RMF, were not included in the analyses. The trajectories described here are generally followed much longer, placing greater constraints on precision. 
The amplitude of the electric field, $\mathbf{E}=-(\partial \mathbf{A} / \partial t) / c$, generated by the RMF is $E \sim$ $\omega_{R} r_{s} B_{R} / c \sim 200 \mathrm{~V} / \mathrm{cm}$ for $B_{R}=20 \mathrm{G}$ and $\omega_{R}=\omega_{c i}$. This field causes ion acceleration and deceleration, $d H / d t=q_{i} \mathbf{E} \cdot \mathbf{v}$, depending on the relative phase of the instantaneous electric field and the particle velocity. Acceleration predominates for low energy particles.

For a typical code run, Fig. 1a) shows deuteron energy $H$ as a function of dimensionless scaled time $\tau=t / \tau_{c i}$, with $\tau_{c i} \equiv 2 \pi / \omega_{c i}$ the ion cyclotron period at the midpoint field, for $B_{R}=20 \mathrm{G}, \mathrm{OMFAC} \equiv \omega_{R} / \omega_{c i}=+0.95$, and $k=\pi / 50$, i.e. $l=1$. The energy remains near $100 \mathrm{eV}$ for $\tau<800$. At $\tau \sim 910$, the energy begins an explosive growth from 400 $\mathrm{eV}$ to $6 \mathrm{keV}$, reached in $\Delta \tau \sim 50$. An enlarged view of this interval is shown in Fig. 1b). The main energy gains occur in steps of half of an RMF period, showing the absence of a sharp resonance. Energy-gaining steps are separated by several RMF periods. This figure also illustrates the high resolution provided by the adaptive integrator. Returning to Fig. 1a), the energy returns to less than $1 \mathrm{keV}$ before growing to $17 \mathrm{keV}$ at $\tau \sim 3.6 \times 10^{3}$, corresponding to $t=0.24 \mathrm{~ms}$. When this trajectory is followed further, to $\tau=8 \times 10^{4}$, the maximum energy rises only slightly, to $19.2 \mathrm{keV}$. We see is no evidence for diffusion to higher energies. The average energy over this run is $6.6 \mathrm{keV}$.

Most (95\%) of the energy is gained from the radial and azimuthal components of the RMF electric field, not from the weaker axial component. As will be shown later, energy gain is strongly correlated with an RMF frequency in the ion cyclotron frequency range, although the strong variation of the confining magnetic field strength and the aperiodic nature of the orbits preclude a sharp resonance. As a result, energy gain is primarily transverse to the confining magnetic field, causing high-energy particles to be well-confined and preferentially localized to the neighborhood of the midplane. Figure 1c) shows the $z$ position of the same orbit as a function of scaled time $\tau$. At early times, $\tau<900$, when the deuteron has low energy, its orbit frequently stagnates near the $z$ extrema of the flux surface, $z \sim \pm 13 \mathrm{~cm}$, due to Speiser collisions (i.e. non-conservation of magnetic moment) and mirror trapping. After the energy grows to $5 \mathrm{keV}$ at $\tau \sim 1000$, the orbit is localized to $|z|<3 \mathrm{~cm}$. The correlation between high energy and small $|z|$ is also seen in Fig. 1d).

Figure 2 presents surveys of the dependences of the maximum deuteron energy on RMF frequency and amplitude. Figure 2a) shows, for $B_{R}=8,32$, and $144 \mathrm{G}$, the maximum energy attained within $\tau=10^{4}$. Figure $2 \mathrm{~b}$ ) shows the variation of maximum energy with $B_{R}$ for fixed $\mathrm{OMFAC}=0.5,1.0,1.5$, and 2.0. Important results are: (1) heating is greatest in the ion-cyclotron range of frequencies, $0.1<|\mathrm{OMFAC}|<1.4$, with the upper limit increasing with $B_{R}$; (2) heating to fusion-relevant energies is independent of RMF rotation direction; (3) only a relatively small amplitude RMF, $\sim 0.1 \%$ of the axial field, is required to heat to fusion-relevant energies; and (4) heating increases strongly above a threshold amplitude of $B_{R}$, which depends on OMFAC. Fig. 2b) is asymmetric about $\mathrm{OMFAC}=0$, with high attained energies extending to higher OMFAC values for positive OMFAC. This is qualitatively consistent with the Doppler-shifted RMF frequency seen by deuterons performing betatron orbits. The sharp boundary to the maximum energy attained at positive OMFAC may be useful for selective heating of $\mathrm{He}_{3}$ in $\mathrm{D}-\mathrm{He}_{3}$ mixtures.

For a heating duration of $\tau=10^{4}$, the scaling of maximum energy attained is found to be approximately $E_{\max } \sim \omega_{R}^{0} B_{a}^{2} B_{R}^{1.5} \kappa^{1 / 2} r_{s}^{2}$ for parameters in the ranges $0.1<|\mathrm{OMFAC}|<$ 
$1,1<\kappa<10,1<r_{s}<40 \mathrm{~cm}, 10^{-4}<B_{R} / B_{a}<10^{-2}$, and $0.1<B_{a}<10 \mathrm{~T}$.

Not all heated ions remain localized near the midplane. If an ion reaches the X-point region, it may be lost. For the RFRC, the loss rate, negligible at $B_{R}=20 \mathrm{G}$, increases markedly above $B_{R}=50 \mathrm{G}$. The self-consistent electrostatic potential, $\varphi$, and alternative magnetic boundary conditions will likely affect losses.

In summary, we have shown that small-amplitude odd-parity rotating magnetic fields can be used in modest-sized FRC devices to heat ions to fusion-relevant energies. The orbits of the high energy ions preferentially localize near the midplane, which could have good implications for stability and fusion reactivity. Moreover, the high-energy-ion orbits are of the betatron type, and may contribute to sustaining the FRC's current. The fully $3-d$ model with strong field gradients we have described should be of considerable interest in the general area of multidimensional chaos. ${ }^{27}$

This work was supported in part by the U.S. Department of Energy Contract No. DE-AC02-76-CHO-3073.

\section{References}

1. For a review, see M. Tuszewski, Nucl. Fusion 28, 2033 (1988).

2. D.C. Barnes, M. Binderbauer, R.D. Brooks, et al., Fusion Technology 30, 116 (1996).

3. M.N. Rosenbluth and M.N. Bussac, Nucl. Fusion 19, 489 (1979).

4. M. Lampe and W. Mannheimer, Naval Research Laboratory Report NRL/MR/6709$98-8305$.

5. N. Rostoker, M. Binderbauer, and H. Monkhorst, Science 278, 1419 (1997).

6. S.A. Cohen and R.D. Milroy, Phys. Plasmas 7, 2539 (2000).

7. H.A. Blevin and P.C. Thonemann, Nucl. Fusion: 1962 Supplement, Part 1, 55.

8. I.R. Jones, Phys. Plasmas 6, 1950 (1999).

9. e.g. R.D. Milroy, Phys. Plasmas 6, 2771 (1999) and references therein.

10. W.N. Hugrass and I. R. Jones, J. Plasma Physics 29, 155 (1983).

11. W.N. Hugrass and M. Turley J. Plasma Physics 37, 1 (1987).

12. D.C. Barnes, J.L. Schwarzmeier, H.R. Lewis, and C.E. Seyler, Phys. Fluids 29, 2616 (1986).

13. L.C. Steinhauer and A. Ishida, Phys. Fluids B2, 2422 (1990).

14. S.A. Cohen, Bull. Amer. Phys. Soc. 44, 586 (1999).

15. For a summary see T.H. Stix, Waves in Plasmas, American Institute of Physics (1992), chapters 11 and 13 .

16. A. Bécoulet, D.J. Gambier, and A. Samian, Phys. Fluids B 3, 137 (1991).

17. L.S. Solov'ev, Rev. Plasma Phys. 6, (1976) 239.

18. For a review see, A.J. Lichtenberg and M.A. Lieberman, Regular and Stochastic Mo- 
tion, Springer-Verlag, NY (1983).

19. M.A. Lieberman and A.J. Lichtenberg, Phys. Rev. A 5, (1971) 1852.

20. J.M. Dawson, "Advanced Fusion Reactors," in Fusion, Vol. B, E. Teller, ed., Academic Press, NY, p. 492 (1981).

21. L.E. Zakharov and V.D. Shafranov, in Rev. Plasma Physics 11, M. Leontovitch, ed., Consultants Bureau, NY (1986), p. 206-211.

22. A. C. Hindmarsh, "ODEPACK, A Systematized Collection of ODE Solvers", in Scientific Computing, R. S. Stepleman et al., Editors (North-Holland, Amsterdam, 1983), pp. 55-64.

23. W.N. Hugrass, J. Plasma Phys. 28, 369 (1982).

24. J.M. Finn and R.N. Sudan, Nucl. Fusion 22, 1443 (1982).

25. For a review, see J. Chen, J. Geophys, Res. 97, 15,011 (1992).

26. T.W. Speiser, J. Geophys. Res. 70, 4219 (1965).

27. G.M. Zaslavskii, M. Yu. Zakharov, A.I. Neishtadt, et al., Sov. Phys. JETP 69, (1989) 885 .

\section{Figure captions}

1. In this figure, color coding is used to distinguish successive time intervals. a) Deuteron energy vs. scaled dimensionless time $\tau=\omega_{c i} t / 2 \pi$. b) Enlarged view of deuteron energy vs. $\tau$ during a period of explosive growth. c) Deuteron axial position $z$ vs. $\tau$. d) Deuteron energy vs. axial position.

2. a) Maximum energy attained by deuterons in the RFRC vs. OMFAC $\equiv \omega_{R} / \omega_{c i}$ for $B_{R}=8,32$, and $144 \mathrm{G}$ and $\tau=10^{4}$. OMFAC was varied in steps of size 0.02 . b) Maximum energy attained by deuterons in the RFRC vs. $B_{R}$ for $\mathrm{OMFAC}=0.5,1.0,1.5$, and 2.0 , and $\tau=10^{4}$. 
PPPL\#00GR006
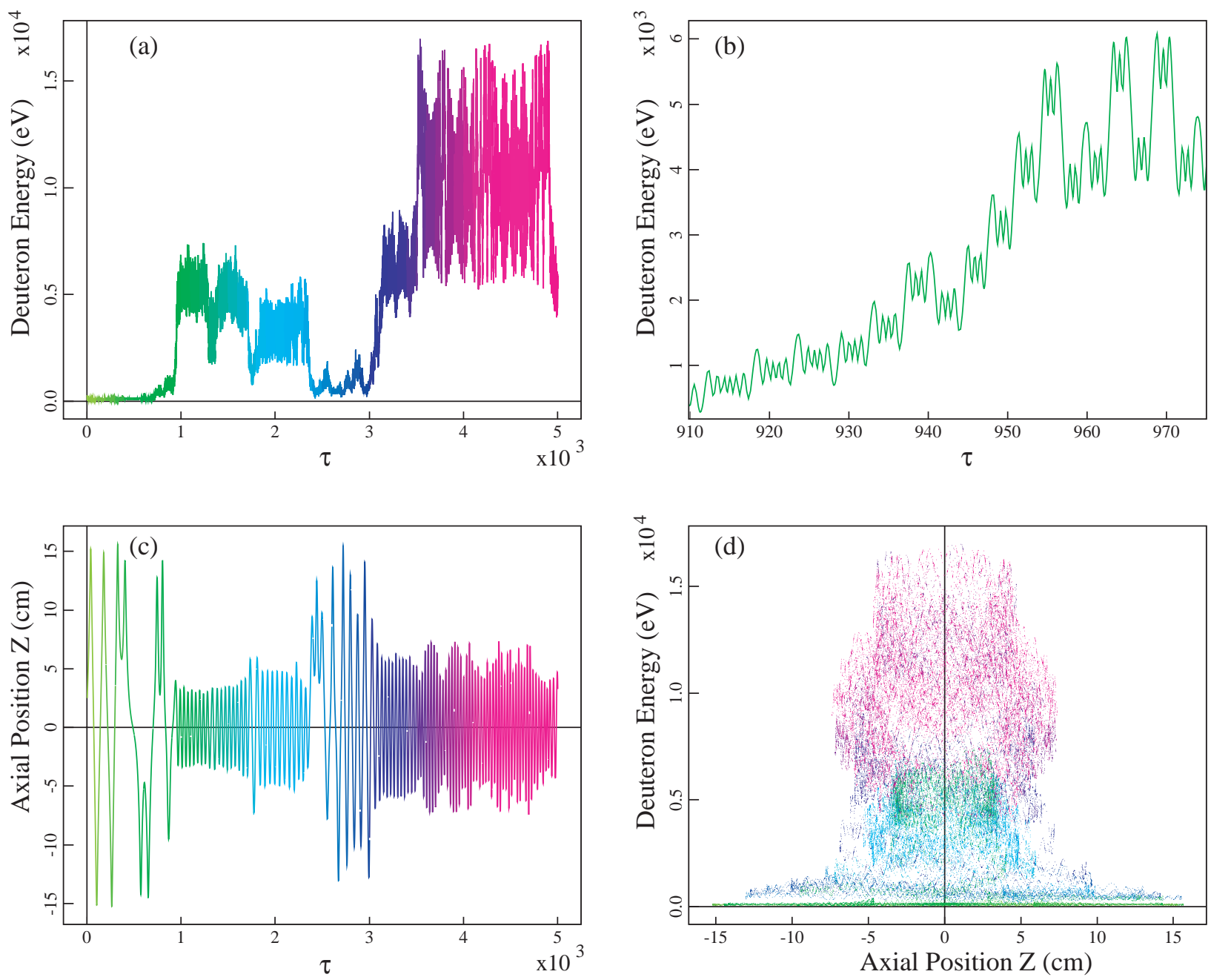
PPPL\#00GR007
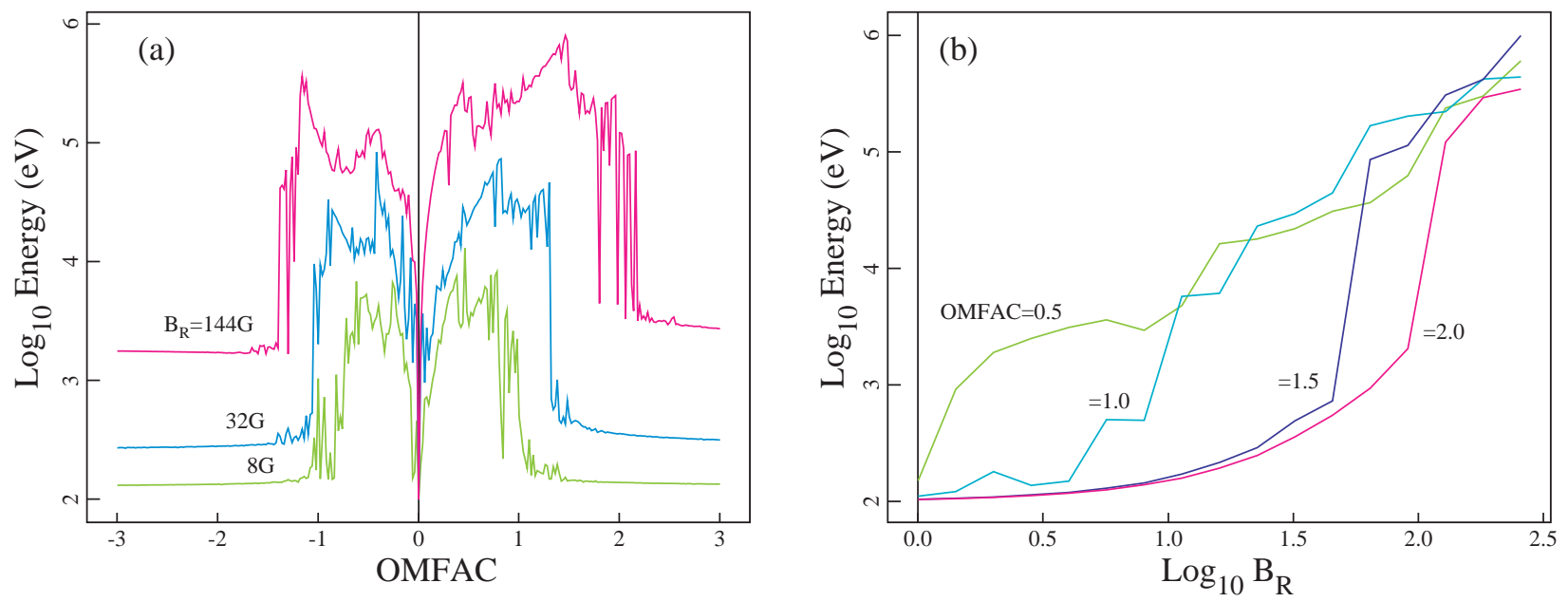
The Princeton Plasma Physics Laboratory is operated by Princeton University under contract with the U.S. Department of Energy.

\author{
Information Services \\ Princeton Plasma Physics Laboratory \\ P.O. Box 451 \\ Princeton, NJ 08543
}

Phone: 609-243-2750

Fax: 609-243-2751

e-mail: pppl_info@pppl.gov

Internet Address: http://www.pppl.gov 